\title{
Analysis of optical density wave propagation and cell movement in the cellular slime mould Dictyostelium discoideum
}

\author{
Florian Siegert and Cornelis J. Weijer ${ }^{1}$ \\ Zoological Institute, University of Munich, Luisenstrasse 14. W-8000 Munich 2. Germany
}

\begin{abstract}
We have studied optical density wave propagation during aggregation of the cellular slime mould Dictyostelium discoideum in a quantitative manner by digital image analysis. The waves are mostly single ended spiral waves starting from an aggregation center. We can measure a variety of parameters such as oscillation frequency, wave propagation velocity and wave shape. This allows the construction of dispersion curves under a variety of experimental conditions. During later development where the optical density waves are no longer visible we have started to measure movement of fluorescently labelled cells. Our main conclusions from these measurements are that the cells continue to move chemotactically to periodic signals both in aggregates and in slugs. There is a dramatic difference in the movement pattern of prestalk and prespore cells: Prestalk cells move perpendicular to the long axis of the slug, they are most likely organized by a scroll wave. Prespore cells seem to move almost perpendicular to the prestalk cells, in the direction of the tip. This hehaviour is explained on the basis of different relay properties of prespore and prestalk cells.
\end{abstract}

\section{Introduction}

During development of the cellular slime mould Dictyostelium discoideum single cells, dispersed over a substratum, aggregate towards an aggregation center. The cells collect in multicellular aggregates $\left(10^{3}-10^{5}\right.$ cells), which transform into a conditional motile stage, the slug. During slug formation the cells start to differentiate into at least two cell types, prespore and prestalk cells. The slug finally transforms into a fruiting body which is composed of two cell types, stalk cells and spore cells $[1,2]$. The aggregation process is brought about by three cellular competences: (1) Periodic production and secretion of cyclic adenosine monophosphate (cAMP) by the cells in the aggregation center. (2) Detection of this signal by cell surface cAMP receptors followed by amplification of the cAMP signal via the activation of adenylate cyclase (relay response). This response is subjected to adaptation which ensures

${ }^{1}$ To whom all correspondence should be sent. outward propagation of cyclic AMP waves. (3) Chemotaxis towards increasing cAMP concentrations. Cells respond to temporal increases in cAMP and therefore cells aggregate unidirectionally in the direction of the aggregation center [3]. Many of the biochemical reactions underlying the cAMP relay response are now known since the dynamics of these reactions can be studied effectively in synchronized cell populations in suspension [2-4]. Based on these data several models have been proposed for the cAMP oscillator [5-7], of which the three-variable model of Martiel and Goldbeter [7] seems to be able to explain the basic features of the oscillator. cAMP wave propagation can be seen as an optical density wave propagation with low-power darkfield optics. The wave impression is caused by a change in the shape and coherence of the cells during their chemotactic response. Chemotactically moving cells are seen as white (light scattering) bands [8]. The optical density waves can be seen to propagate outward from the aggregation center. The patterns seen are either concentric waves or spirals $[9,10]$. The quantitative study of aggregation 
dynamics in vivo has recently become possible by the use of digital image analysis [11].

During later development all morphogenetic movements are organized by the tip, a distinct morphological structure, which is present from late aggregation onwards. The tip behaves as an organizer because it will induce a secondary axis and take over part of the tissue behind it to form a new secondary slug when it is grafted into the side of another slug [12]. The tip also inhibits the formation of new tips [13]. Many of the properties of the tip can be explained by assuming that it is a pacemaker for cAMP signals. The test of this hypothesis is however not as straightforward as it might seem, since in these later structures darkfield waves are no longer visible. The darkfield waves can only be seen for about 20 periods during early development and disappear when the cells start to make aggregation streams [9-11]. The goal of the work described below is to compare quantitative measurements of optical density wave propagation (spreading of the signal) and of cell movement (the cell response). Analysis of cell movement is currently the only way to deduce the pattern of signal propagation in aggregates and slugs.

\section{Results}

\subsection{Analysis of optical density wave propagation during early aggregation}

We have studied several parameters associated with the propagation of optical density waves during the initial stages of aggregation. In general there are around 20-25 waves emanating from an aggregation center. The waves can be concentric, but with our strains and experimental conditions more than $95 \%$ of the waves form spirals (fig. 1A). Almost all spirals have only one arm (fig. 1A), but on rare occasions unstable double armed spirals can be observed (figs. 1B, 1C). We have developed a technique based on digital image analysis with which we can easily measure oscilla- tion frequency, wave propagation speed, wave shape and amplitude of propagating optical density waves [11]. In wild type cells it is seen that the oscillation frequency increases during development (period length decreases from 6 to $3 \mathrm{~min}$ ), while the propagation speed decreases from 600 to $300 \mu \mathrm{m} / \mathrm{min}[10,11]$. From these data one can construct a dispersion curve (relationship between wave propagation velocity and period length, open circles in fig. 2) that fits well with model calculations based on the MartielGoldbeter model [14].

\subsection{The relay inhibitor caffeine induces biphasic waves}

By disturbing the normal kinetics of the oscillation with the aid of mutations or drugs it will be possible to investigate which variables are important for wave formation and stable propagation. We have investigated the effect of caffeine (an inhibitor of adenylate cyclase activation [15]) on wave formation and propagation and found that inhibition of the cAMP relay response (cAMP signal strength) leads to fewer aggregation centers and slower oscillating and slower propagating waves [11]. This is reflected in a widely different dispersion relation as compared to the control case (fig. 2). Since the form of the dispersion relation is mainly dependent upon the chemical kinetics of the oscillator it will be of interest to see how these findings can be accommodated in models for the cAMP oscillator. Since it is now possible for the first time to obtain experimental data rather easily and with good accuracy it offers the possibility for a fruitfull interaction between experiment and theory $[11,14]$.

\subsection{Biphasic waves}

We have found biphasic waves under a variety of experimental conditions. They can be very clearly seen in cells aggregating on the relay 

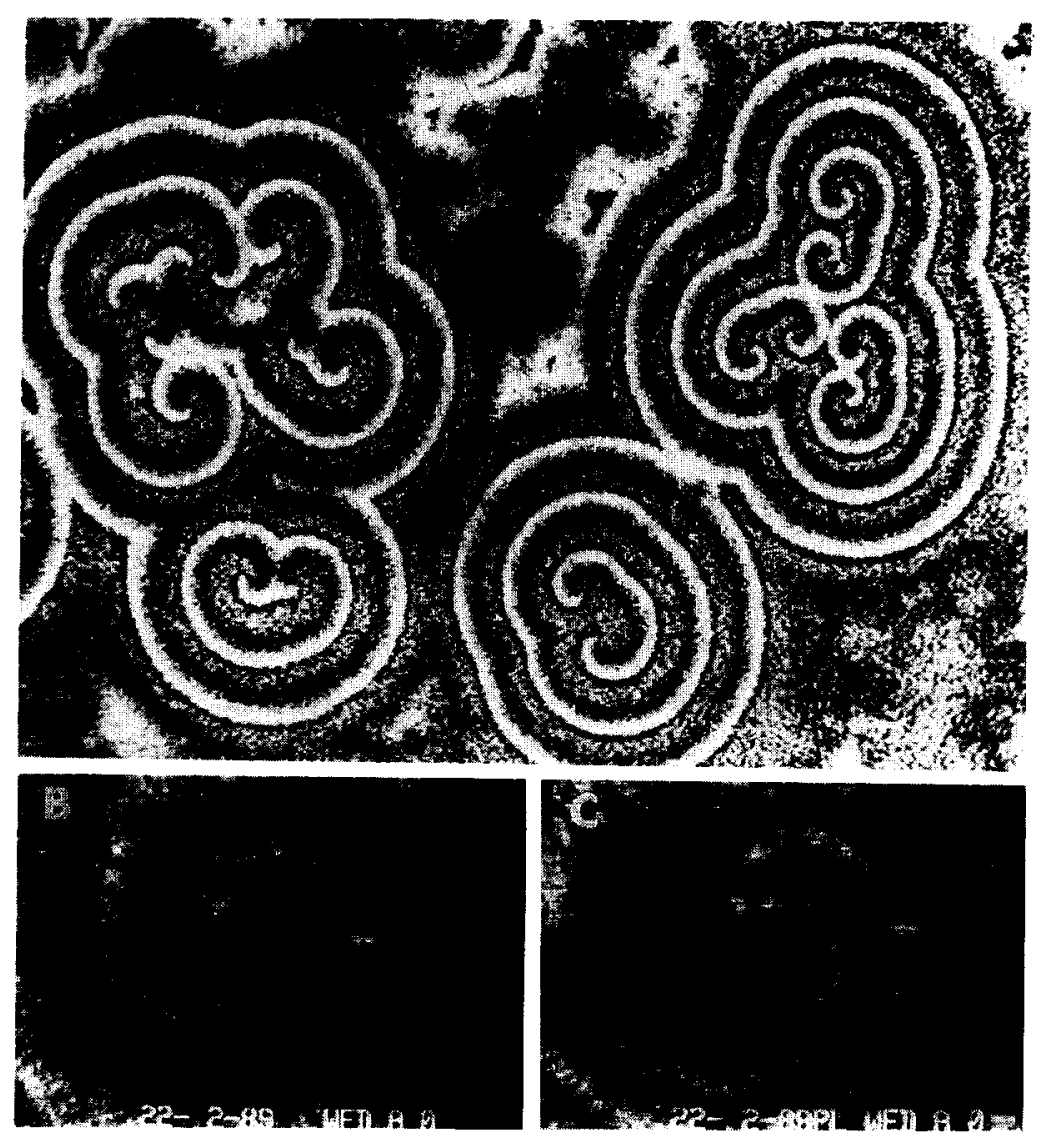

Fig. 1. (A) Low-power view of optical density waves in a field of aggregating AX-2 cells. The appearance of the waves was enhanced by real time image differencing between all incoming waves and an initial reference frame. This procedure detects changes from a reference time point and indicates motion. (B) High-power darkfield photograph of a double armed spiral. (C) High-power photograph of the same spiral shown in (B), 13 min later. The double armed spiral is unstable since one arm overtakes the other, which leads to extinction of both arms.

inhibitor caffeine (fig. 3). Analysis of the power spectrum shows that these signals are composed of two harmonic frequencies [11].

Since the optical density signal consists of the response of many cells we believe that the wave form may reflect a heterogeneity in the response of the cells. The presence of discrete response elements (cells) with variable properties distinguishes this system from more homogeneous chemical systems. Not all cells can make a response every time that they are hit by a signal since they are not yet completely deadapted. This leads to a partial gating of signals coming from the aggregation center, i.e. some cells can re- spond to every signal while others with an intrinsic longer deadaptation time can only respond to every second pulse. This gating can happen either at the level of the relay response or at the level of the chemotactic response $[11,16]$. If gating occurs at the level of the chemotactic response this will lead to a sorting out of fast and slow responding cells. Fast cells will end up in the tip, thereby creating an inhomogenous excitable medium. In order to distinguish between these two alternative hypotheses we will have to measure the rate of movement of individual cells under conditions where the optical density signals are mono- and bi-phasic. 


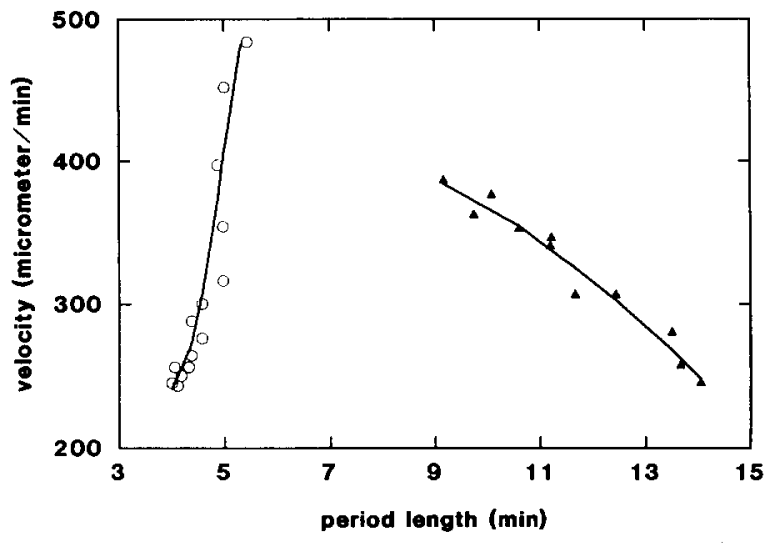

Fig. 2. Relationship between wave propagation velocity and period length (dispersion relation) of successive waves during aggregation. The dispersion relation is shown for wild type AX-2 cells (open circles) and AX-2 cells aggregating on $5 \mathrm{mM}$ caffeine (solid triangles). The data shown are the mean values derived from more than 40 aggregation centers in 15 independent experiments. Wave propagation velocity and period length were determined by digital image processing as described in ref. [11].

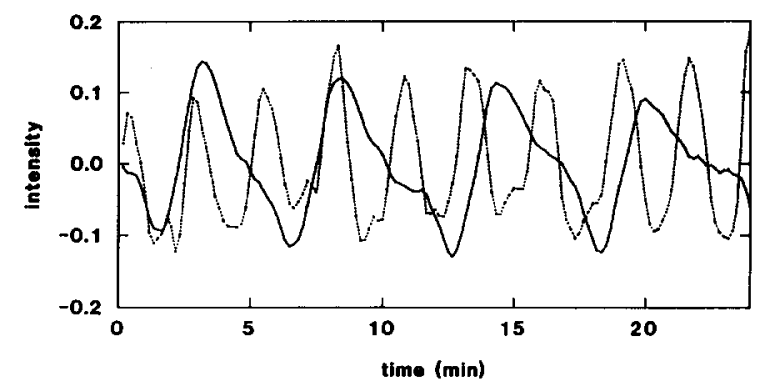

Fig. 3. (A) Optical density oscillation of AX-2 cells showing regular oscillations (dotted line) and of cells aggregating on $5 \mathrm{mM}$ caffeine showing biphasic oscillations (solid line). For methods see ref. [11].

\subsection{Analysis of movement of single cells}

As a first step we have measured the movement of individual cells aggregating to an aggregation stream. We have chosen fluorescent tagging of the cells since this allows us to follow individual cells in multicellular stages of development, such as in aggregation streams, moulds and slugs. We can detect and follow several labelled cells every few seconds anywhere in a video frame and save the grey level information of all the pixels of the cell and a small surrounding square. This allows us to follow the cell shape and fluorescence intensity changes while it is moving. Simultaneously we can calculate several parameters like rate and direction of movement and changes in cell shape. This is a procedure which will also allow us in the near future to correlate changes in physiological variables during chemotaxis using the newly developed fluorescent ion specific membrane potential sensitive dyes.

As can be seen the rate of movement of these cells is highly periodic and can be easily measured (fig. 4).

\subsection{Movement of cells in aggregation streams}

To test whether it is possible to measure periodic cell movement in streams we have measured movement rates of individual cells in aggregation streams of streamer mutants. Streamer mutants form long aggregation streams in which one can see the propagation of optical density waves (figs. $5 A, 5 B)[11,16,17]$. These waves arise by the local accumulation and depletion of cells and reflect differences in stream diameter. As can be seen in fig. 5C individual cells do move periodically although the periodicity is not as clear as in the darkfield waves. This must be attributed to the fact that we are now looking to the response of an individual cell and not to a population response as in the case of darkfield waves. However, power spectrum analysis indicates a clear periodicity of cell movement of approximately 2.5 min (not shown).

\subsection{Movement of cells in slugs}

We have performed similar measurements in slugs and it appears that the movement of single cells in slugs is at least as periodic as that of cells in aggregation streams (fig. 6B). Furthermore it can be seen that the cells change their shape in a characteristic fashion during the periodic move- 


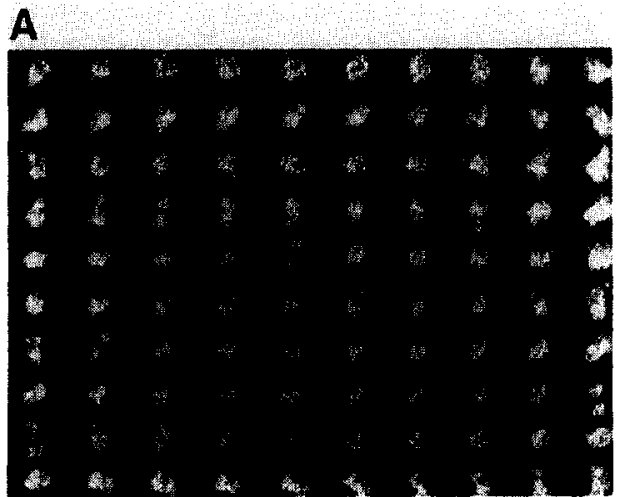

B

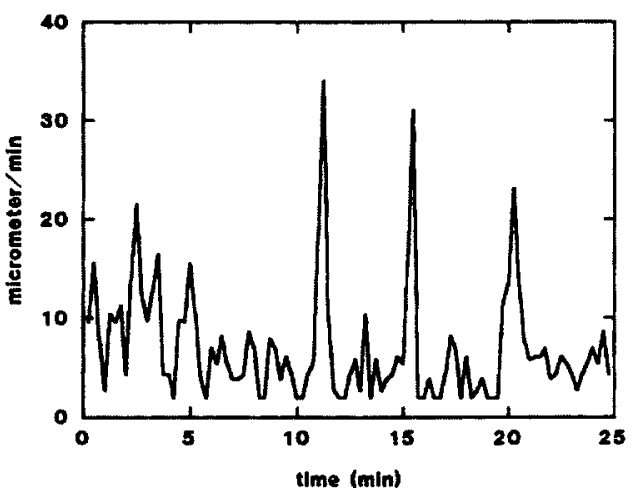

Fig. 4. Movement of a single cell towards an aggregation center. (A) Successive frames showing the shape changes of a single cell aggregating towards an aggregation center. The cell was labelled with a rhodamine dextran (MW 10000 D) conjugate that was brought in the cell by filter loading. The cells to be loaded were pressed through a $7 \mu \mathrm{m}$ Nythal filter (Schweiz. Seidengazefabrik AG Thal) in the presence of rhodamine dextran. Under the conditions employed about $1 \%$ of the cells were labelled and retained the label for more than $24 \mathrm{~h}$. Fluorescence was detected by a Hamamatsu silicon intensified target (SIT) camera (C-2400-8) and the resulting image digitized with $512 \times 512$ pixel resolution. Cells were detected by recording intensity values over a certain threshold and the center of mass was calculated. A window of $50 \times 50$ pixels around the center of the cell was saved on a hard disk. This procedure was repeated every 10 or $15 \mathrm{~s}$. From these data a new picture was constructed by placing all consecutive images in rows behind each other. The top left image is the first image and the bottom right image is the last. (B) Rate of movement of the cell seen in (A). The movement was calculated on the basis of the displacement of the center of the cell as described in (A). Every data point corresponds to a picture in (A).

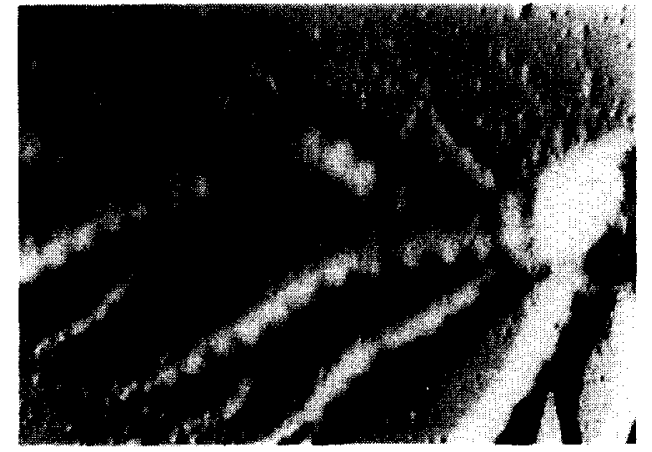

B

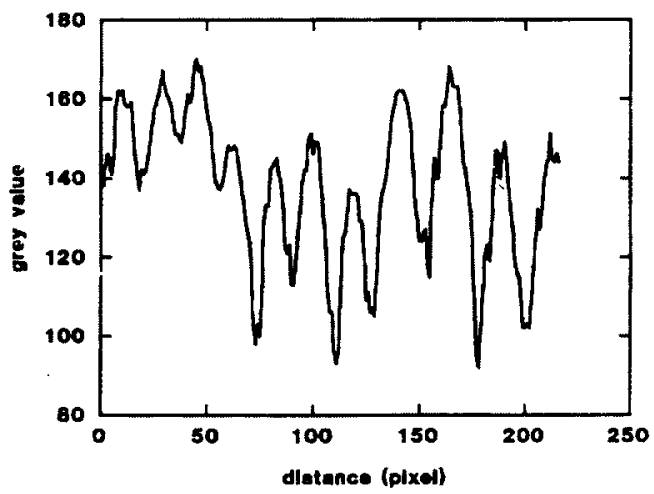

C

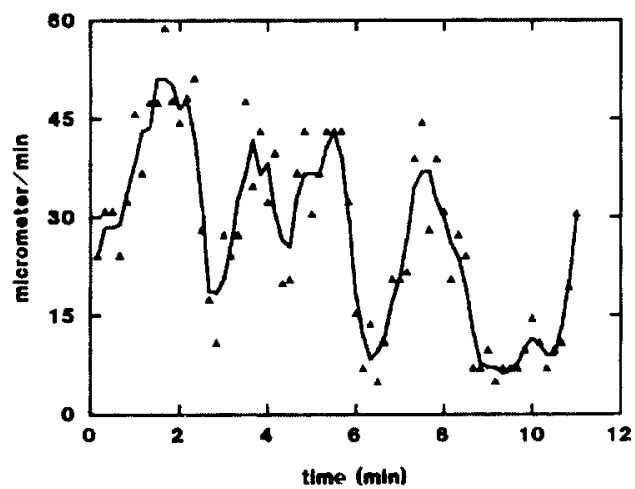

Fig. 5. Optical density wave propagation and cell movement in aggregation streams of streamer mutant NP377. (A) Digitized image of aggregation streams of the streamer mutant NP377. (B) Optical density waves measured along the black line through the aggregation stream shown in (A). (C) Rate of movement of a fluorescently labelled cell in such an aggregation stream. The rate of movement was determined as described in the legend of fig. 4. The solid line was calculated by moving average smoothing over three neighbours. The original data are shown as solid triangles. 


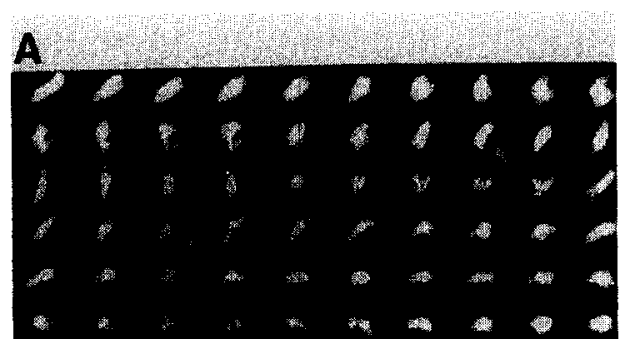

B

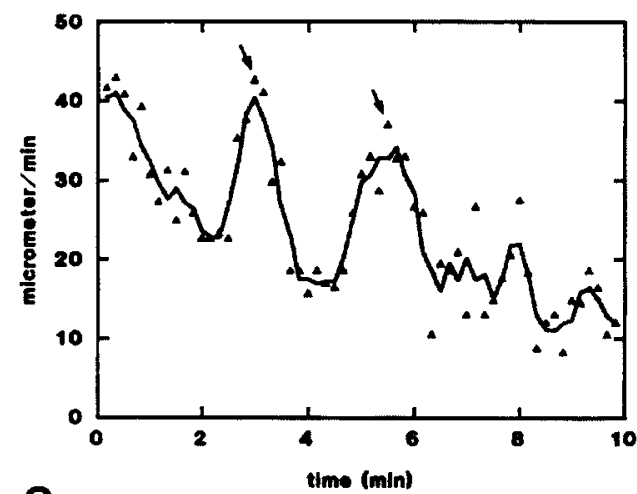

C

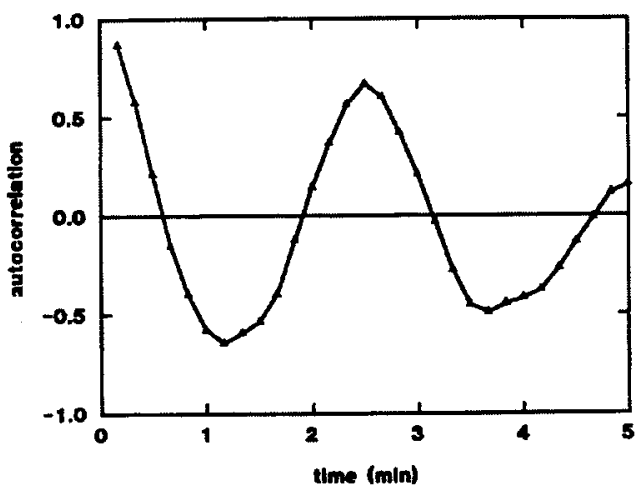

Fig. 6. Movement of a fluorescently labelled cell in the prespore zone of a slug (AX-2). (A) A time series of images showing the successive shape changes of the cell. Notice the periodic change in cell shape. If the cell is moving fast it is elongated, when it is moving slow it extends pseudopods. Two successive time points where the cell moves fast are indicated by white arrows in the second and the fourth row of the time series. (B) Rate of movement of the cell shown in (A). Two time points where the cell is elongated (two white arrows in (A)) are indicated by two black arrows. This shows that elongated cells move fast. Methods as described in fig. 4. The solid line was calculated by moving average smoothing over three neighbours. The original data are shown as solid triangles. (C) Autocorrelation of data in (B) shows that the cell movement is clearly periodic, with a for slugs typical period length of $2.5 \mathrm{~min}$. ment. At the times of slow movement they stick out pseudopods in different directions before they decide to continue in one or to proceed in the going direction (fig. 6A). This is a behaviour that is well known from cells making chemotactic movement and shows that cells in slugs move in a chemotactic fashion.

\subsection{Global analysis of movement in aggregates and slugs}

Since a slug consists of $10^{3}-10^{5}$ cells it is rather difficult to extract data about the behaviour of the cell population by the above mentioned methods. Therefore we have resorted to a technique that allows us to map the movement of many cells in a slug simultaneously. In a slug that contains many labelled cells, we can detect which cells have a fluorescence above a certain threshold and keep track of the times that pixels are above threshold. If this information is displayed one obtains tracks where cells have moved. Additionally we keep track how many times a pixel at a certain location is above threshhold. This is displayed in a 16-valued red scale from black to bright red whereby bright red indicates that a pixel has been above threshhold 16 times or more. Therefore if a cell moves slowly the resulting track appears bright red at this location and if the cell is moving fast the track appears dark red or black (plates I and II). This method allows us to track fluorescently labelled moving cells in real time and to display this information in an easily interpretable way.

To test the method we have followed several labelled cells in an AX-2 aggregate. As can be seen (plate IA) cells in streams move periodically in the direction of the aggregation center, while the cells in the body of the aggregate move in a spiral fashion around a central core. The movement of the cells is indicated in the lower graphical representation of the results (plate IB).

We have observed spiral movement in aggregates and mounds in many experiments (results will be published in detail elsewhere). Now the 

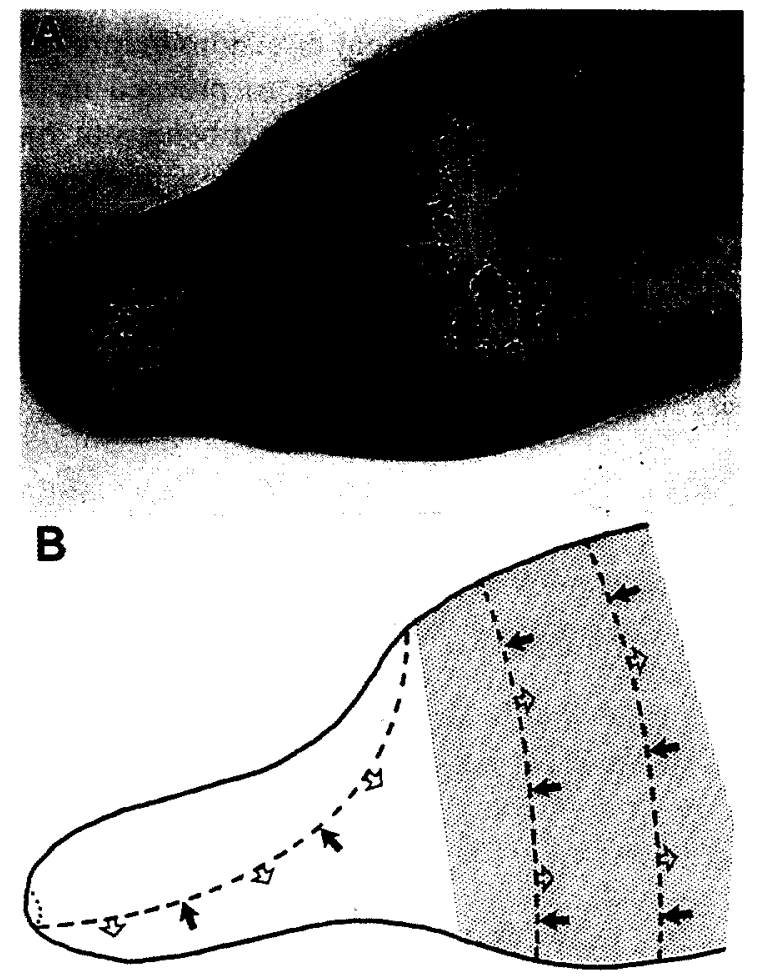

Plate I.
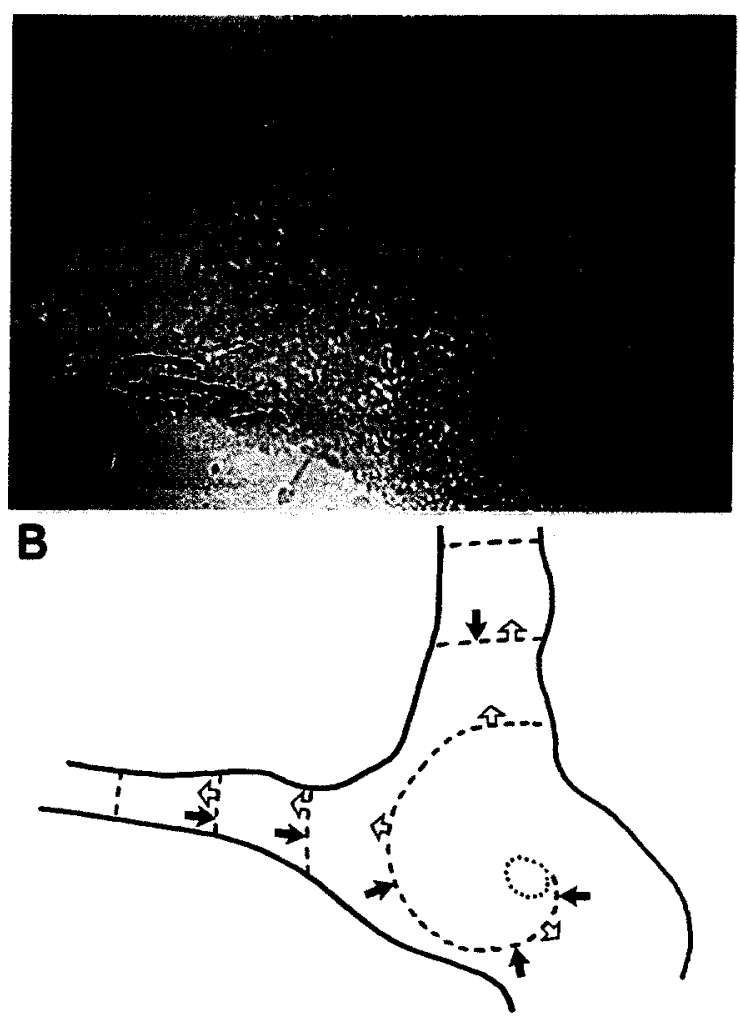

Plate II.

Plate I. Direction and rate of movement of several cells in an aggregate. (A) Cells were fluorescently labelled as described in fig. 4. The image of the aggregate was digitized and a threshold was set such that all the intensity values of fluorescent cells were above the threshold. The position of all pixels above threshold was saved in the memory of our video digitizer board and given the value 1 . This procedure was repeated for successive times and when the grey level of a pixel above threshold had been above threshold previously the value of this pixel was incremented by 1 . This procedure leads to the formation of tracks which show where the cells have moved. The tracks are shown superimposed on the original picture. The value of a given pixel in the track is color coded in a 16-valued red scale. Black means occupied only once (fast movement) and bright red means occupied 16 times or more (slow or no movement). It can clearly be seen that the cells in the aggregation stream move periodically in the direction of the center while the cells in the aggregation center move more continuously in spirals. (B) Schematic diagram of observed cell movement (black arrows) and the thereof derived propagation of waves. The wave fronts are indicated as broken lines, the direction of propagation is indicated by the open arrows.

Plate 1I. Movement of cells in a slug. (A) Several fluorescently labelled cells both in the prespore and prestalk zone of a slug are followed with the method described in plate I. Cells in the prespore zone move in the direction of the tip, while cells in the prestalk zone move perpendicular to the slug tips main axis. (B) Schematic diagram depicting cell movement in slugs and the derived propagation of the waves. The direction of cell movement is indicated by the black arrows, the wave fronts are indicated by the broken lines and the direction of wave propagation by the open arrows. 
interesting question is what happens in slugs? As can be seen in plate IIA cells in the prestalk zone of slugs move in a direction almost perpendicular to the long axis of the slugs, while cells in the back (prespore zone) of the slug move in a direction parallel to the long axis of the slug. Since the chemotactic movement in the prestalk zone is perpendicular to the propagating wave front, this implies that the wave front must extend along the long axis of the slug tip. Therefore the slug tip is a scroll wave which decomposes in waves traveling almost perpendicular to the long axis of the slug in the prespore zone. This situation is depicted schematically in plate IIB. These observations are not compatible with the inverse fountain flow model for slug movement as proposed recently [18], but are compatible with the observations of movement of neutral red stained anterior-like cells in the prespore zone of slugs [19, 20].

This change in the geometry of the propagating waves is in our opinion most likely caused by a difference in the oscillatory properties of the cells in the prestalk and in the prespore zone. It is well documented that prespore and prestalk cells sort out from each other when mixed experimentally and that this sorting out is due to chemotaxis [21, 22]. This opens in principle the possibility that one collects cells with different oscillatory properties in the prestalk and prespore zone of the slug. We have previously shown that one can separate aggregation stage cells that will sort to the tip of the slug from those that will sort to the back of the slug. The cells that will sort to the front have higher intrinsic oscillation frequencies than cells that will sort to the back of a slug [23]. Furthermore cells in the prespore zone can break-down the external cAMP less efficiently as the cells in the prestalk zone since they have less phosphodiesterase [24]. This would leave them always being partly adapted. Since they are partly adapted they will oscillate slower and the amplitude of the released signal will be smaller than that from the prestalk cells. The result of these differences will be that cells that are in the tip are those cells that will be able to oscillate and chemotax rapidly.
They will move around in a slightly tilted scroll wave [25] along the tip long axis. The other cells outside the tip cannot propagate the cAMP signal anymore around the tip axis, which will be the minimal refractory period attainable by a spiral wave. The end of the scroll wave however will be a continuous source of cAMP which can trigger new waves in the prespore zone which can then move along the slug main axis. Waves will be able to propagate either along the slug main axis or as slowly winding spirals depending on the diameter of the prespore piece.

It is clear that there has to be a supercellular control mechanism to coordinate the behaviour of a mass of single cells in the slime mould. On the basis of the experiments described above, we believe that the tissue is an excitable threedimensional medium, and that morphogenesis must be based on slight local changes in signal propagation and chemotactic responses. An extra complication in the description of this system is that the responding units (cells) can change their neighbours and rearrange themselves in new patterns, contributing to spatial heterogeneity. This introduces an extra feedback element into the system that will make it even more interesting to study. We are looking forward to a comparison of this intuitive explanation with three-dimensional model calculations for example based on cellular automata [26].

\section{Acknowledgements}

We like to thank Dr. P.C. Newell (Oxford) for his gift of the streamer F mutants and Dr. H.K. MacWilliams for critically reading the manuscript. This work was supported by a grant from the Deutsche Forschungsgemeinschaft to C.J. Weijer (We 1127).

\section{References}

[1] W.F. Loomis, Dictyostelium discoideum, A Developmental System (Academic Press, New York, 1975). 
[2] R.H. Kessin, Microbiol. Rev. 52 (1988) 29.

[3] P.N. Devreotes, Adv. Cycl. Nucl. Res. 15 (1983) 55.

[4] P.C. Newell, G.N. Europe-Finner and N.V. Small, Microbiol. Sci. 4 (1987) 5.

[5] H. Meinhardt, Differentiation 24 (1983) 191

[6] L.A. Segel, A. Goldbeter, P.N. Devreotes and B.E. Knox, J. Theor. Biol. 120 (1986) 152.

[7] J.L. Martiel and A. Goldbeter, Nature 313 (1985) 590.

[8] F. Alcantara and M. Monk, J. Gen. Microbiol. 85 (1974) 321.

[9] A.J. Durston, Dev. Biol. 37 (1974) 225.

[10] J. Gross, M. Peacy and D. Trevan, J. Cell Sci. 22 (1976) 645.

[11] F. Siegert and C.J. Weijer, J. Cell Sci. 93 (1989) 325.

[12] J. Rubin and A. Robertson, J. Embryol. Exp. Morphol. 33 (1975) 227.

[13] H.K. MacWilliams, Symp. Soc. Devel. Biol. 40 (1984) 463.

[14] J.J. Tyson and J.D. Murray, Development 106 (1989) 421.

[15] M. Brenner and S. Thoms, Dev. Biol. 101 (1984) 136.
[16] K. Gottmann and C.J. Weijer, J. Cell Biol. 102 (1986) 1623.

[17] F.M. Ross and P.C. Newell, J. Gen. Microbiol. 127 (1981) 339.

[18] G.M. Odell and J.T Bonner, Phil. Trans. R. Soc. London BM12 (1986) 487.

[19] A.J. Durston and F. Vork, J. Cell Sci. 36 (1979) 261.

[20] R.L. Clark and T.L. Steck, Science 204 (1979) 1163

[21] S. Matsukuma and A.J. Durston, J. Embryol. Exp. Morphol. 50 (1979) 243.

[22] J. Sternfeld and C.N. David, Differentiation 20 (1982) 10

[23] C.J. Weijer, S.A. McDonald and A.J. Durston, Differentiation 28 (1984) 9.

[24] A.P. Otte, M.J.E. Plomp, J.C. Arents, P.M.W. Janssens and R. van Driel, Differentiation 32 (1986) 185.

[25] A.T. Winfree, When Time Breaks Down (Princeton Univ. Press, Princeton, NJ, 1987).

[26] M. Gerhardt, H. Schuster and J.J. Tyson, Science 247 (1990) 1563. 